\title{
Virtual Reality als Trainingsmethode: Eine Laborstudie aus dem Industriebereich
}

\author{
Josef Wolfartsberger (D) - René Riedl · Herbert Jodlbauer • \\ Niklas Haslinger • Andrii Hlibchuk • Alexander Kirisits • Stefan Schuh
}

Eingegangen: 15. Juli 2021 / Angenommen: 17. November 2021 / Online publiziert: 14. Dezember 2021

(C) Der/die Autor(en) 2021

Zusammenfassung Virtual Reality (VR) gilt als vielversprechende Technologie, nicht zuletzt deshalb, weil damit komplexe Inhalte vermittelt werden können. Da VR ein hohes Potenzial für Interaktivität und Immersion aufweist, findet die Technologie auch in der Industrie Anwendung, beispielsweise für die realitätsnahe Simulation von Montage- und Instandhaltungsarbeiten. Wissenschaftliche Befunde zur Wirksamkeit des Lernens in industriellen VR-Umgebungen existieren bislang kaum. Es ist daher auch unklar, wie Lerneffekte in virtuellen Umgebungen verbessert werden können. Der vorliegende Artikel untersucht, ob VR-gestütztes Training im Vergleich zu klassischem training-on-the-job (begleitet von einem Tutor) zu einer Steigerung des Lernerfolgs führt. Basierend auf einem VR-Trainingstool zum Erlernen von Montageabläufen wurde eine Laborstudie mit 24 ProbandInnen durchgeführt. Die Ergebnisse zeigen, dass VR-gestütztes Training zwar als weniger anstrengend empfunden wurde, der Lernerfolg jedoch im traditionellen training-on-the-job signifikant besser ausfiel. Auf Basis dieser Erkenntnisse werden die Chancen und Risiken aktueller VR-Trainingssimulationen diskutiert und konkrete Handlungsempfehlungen zur Steigerung des Lerneffekts formuliert.

Schlüsselwörter Virtual Reality (VR) · Lernumgebung · Training · Industrie · Laborstudie

Josef Wolfartsberger $(\bowtie) \cdot$ Herbert Jodlbauer · Niklas Haslinger · Andrii Hlibchuk ·

Alexander Kirisits · Stefan Schuh

Fakultät für Wirtschaft \& Management, Center of Excellence for Smart Production, Fachhochschule Oberösterreich, Wehrgrabengasse 1-3, 4400 Steyr, Österreich

E-Mail: josef.wolfartsberger@fh-ooe.at

René Riedl

Fakultät für Wirtschaft \& Management, Abteilung für Digital Business, Fachhochschule

Oberösterreich, Wehrgrabengasse 1-3, 4400 Steyr, Österreich

Institut für Wirtschaftsinformatik - Information Engineering, Johannes Kepler Universität Linz, Altenberger Straße 69, 4040 Linz, Österreich 


\title{
Virtual Reality as Training Method: A Laboratory Study in the Industrial Domain
}

\begin{abstract}
Virtual reality (VR) is considered a promising technology because it can be used to convey complex contents. Since VR has a high potential for interactivity and immersion, the technology is also used in industry, for example for the realistic simulation of assembly and maintenance work. However, in this context there are hardly any scientific findings on the effectiveness of learning in VR environments. It is also unclear how learning can be improved in virtual environments. This article investigates if VR-supported training leads to an increase in learning success compared to classic training-on-the-job (accompanied by a tutor). Based on a VR training tool for learning assembly processes, a laboratory study was conducted with 24 participants. The results show that although VR-supported training was perceived as less strenuous, the learning success (measured with assembly time and error rate) was significantly better in traditional training-on-the-job. Based on these findings, the opportunities and risks of current VR training simulations are discussed and recommendations for action to increase the learning effect are formulated.
\end{abstract}

Keywords Virtual Reality (VR) · Learning Environment · Training · Industry · Laboratory Study

\section{Einleitung}

Virtual und Augmented Reality-(VR/AR)-Hardwaresysteme entwickeln sich rasant. Die Steigerung der Rechenleistung und die hohe Verfügbarkeit von Endgeräten tragen dazu bei, dass viele Unternehmen über mögliche Einsatzszenarien im betrieblichen Umfeld nachdenken und diese auch umsetzen (Zenisek 2021). Besonders im Bereich des industriellen Trainings zeigt sich, dass VR-Systeme komplexe Situationen realistisch nachbilden können. Interaktivität und Immersion versprechen eine gesteigerte Motivation der Lernenden und auch einen höheren Lernerfolg bei gleichbleibenden oder niedrigeren Kosten (Abulrub et al. 2011). Bis heute gibt es in diesem Kontext jedoch kaum Studien, die den möglichen positiven Lerneffekt empirisch belegen. Es zeigt sich, dass zwar virtuelle Trainings existieren, deren Wirksamkeit hinsichtlich Wissensvermittlung jedoch unzureichend erforscht ist.

Der vorliegende Artikel widmet sich der Frage, ob VR-gestütztes Training im Vergleich zu klassischem training-on-the-job (begleitet von einem Tutor) zu einer Steigerung des Lernerfolgs führt. Basierend auf einem VR-Trainingstool zum Erlernen komplexer Montage- und Wartungsabläufe wurde am Campus Steyr der Fachhochschule Oberösterreich eine Laborstudie mit 24 ProbandInnen durchgeführt. In einem fiktiven Szenario mussten die TeilnehmerInnen der Experimentalgruppe ein Objekt unter Anleitung eines Tutors korrekt montieren und nach einer Pause selbiges Objekt eigenständig aus dem Gedächtnis erneut zusammensetzen. Das Training fand in einer komplett virtuellen 3D-Umgebung mit visuellen Hinweisen statt. Beim eigenständigen Zusammenbau im zweiten Durchgang wurden die Montagezeit je Arbeitsschritt, die Fehlerrate sowie die kognitive Belastung bei der Tätigkeitenaus- 
führung mittels NASA-TLX Fragebogen erhoben. Auf der Basis eines BetweenSubjects-Designs trainierte die Kontrollgruppe - auch angeleitet von einem Tutor am realen Objekt. Die Ergebnisse der Studie zeigen, dass die ProbandInnen der Experimentalgruppe das Training in VR als weniger kognitiv belastend wahrgenommen haben. Die Studie zeigt jedoch weiter, dass die ProbandInnen der Kontrollgruppe im zweiten Durchgang das Objekt schneller montieren konnten und dabei auch weniger Fehler machten; zudem benötigten sie weniger Hinweise von einer Drittperson, um die Aufgabe zu erledigen. Daraus lässt sich ein größerer Lernerfolg beim klassischen training-on-the-job schließen.

Der vorliegende Artikel diskutiert die Erkenntnisse dieser Laborstudie und gibt einen Einblick in die Chancen und Risiken von VR für industrielle Trainingsszenarien. Basierend auf Vorprojekten im Bereich VR-gestütztes Training beschreiben wir zunächst Grundlagen des Lernens in der virtuellen Realität und danach skizzieren wir die technische Implementierung des Prototyps für immersives Training bei komplexen Montageprozessen. Dieser Prototyp war Grundlage der Laborstudie. Es folgt die Darstellung der empirischen Studie, um darauf aufbauend die Chancen und Risiken aktueller Implementierungen zu diskutieren und konkrete Handlungsempfehlungen zu formulieren.

\section{Lernen in der virtuellen Realität}

Mit dem Voranschreiten innovativer Technologien sind neue Möglichkeiten der Wissensvermittlung entstanden. VR als Visualisierungstechnologie bietet die Basis für neue Wege der Wissensvermittlung. Erste Erkenntnisse belegen, dass mögliche positive Lerneffekt in VR-Umgebungen mit einem Immersionsgefühl einhergehen (Huang et al. 2021). Demnach ermöglicht VR, vollständig in den Inhalt ,einzutauchen“ und diesen interaktiv zu erleben (Cmentowski et al. 2019). Im Gegensatz zu klassischen Lernmedien (wie Videos oder Büchern) soll VR die Distanz zwischen Rezipienten und Erlebnis verringern, da die Inhalte realitätsgetreu wahrgenommen werden, zumindest aber nahe an die Primärerfahrung herankommen.

Es existieren bereits einige Arbeiten, die sich mit dem Potenzial von VR zur Wissensvermittlung auseinandersetzen. Pirker et al. (2018) beschreiben beispielsweise eine immersive VR-Umgebung zur Vermittlung von Inhalten im Physikunterricht. Die AutorInnen berichten in ihrer Studie von positiven Lernerfahrungen der TeilnehmerInnen, Inhalte wurden als interessant und kurzweilig empfunden. Fromm et al. (2021) haben im Kontext der Wissensvermittlung im Hochschulbereich innovative VR-Prototypen entwickelt und erprobt. Konkret wurde untersucht, wie VRAnwendungen im Bildungsbereich gestaltet werden sollten, um vier unterschiedliche Lernmodi zu unterstützen (im englischen Original als ,,concrete experience, reflective observation, abstract conceptualization, active experimentation" bezeichnet). In der Industrie wird VR häufig in der Produktentwicklung oder im Training von Montage- und Instandhaltungstätigkeiten eingesetzt. Grassini und Laumann (2020) berichten in einem Literatur-Review, dass VR-Training dabei helfen kann, das Personal auf reale Notfallszenarien vorzubereiten. Es wird argumentiert, dass der Einsatz von VR nicht nur das Risikobewusstsein der MitarbeiterInnen verändert, sondern auch 
ihr Routineverhalten positiv beeinflusst. In Summe trage dies zu einer verbesserten Sicherheitskultur bei. Die Autoren verweisen jedoch auch auf die häufig noch bestehende Unausgereiftheit der Technologie und äußern unter Bezugnahme auf andere Arbeiten Zweifel an der Wirksamkeit von VR-Trainings. Ein akutelles Beispiel eines VR-Trainings beschreibt Mulders (2020). Die Arbeit präsentiert die Ergebnisse einer Laborstudie mit 14 angehenden FahrzeuglackiererInnen, die virtuell geschult wurden. Die Ergebnisse zeigen, dass VR-Trainings für die TeilnehmerInnen motivierend sein können, unter anderem beispielsweise wegen der Interaktion mit einem virtuellen Tutor.

Trotz der Verfügbarkeit entsprechender VR-Technologien sowie einiger empirischer Forschungsergebnisse setzen viele Industrieunternehmen nach wie vor auf traditionell bewährte Methoden des Trainings, insbesondere auf training-on-thejob mit einem Tutor, der die Einlernphase begleitet. Aus wissenschaftlicher Sicht interessant ist ein Vergleich von traditionellen Methoden mit neuartigen Formen des Lernens. Pratticò und Lamberti (2021) berichten Ergebnisse einer Studie, in der untersucht wurde, wie ein immersives VR-Training für die Schulung von Industrieroboter-BedienerInnen im Vergleich zu der vom Unternehmen angewendeten Trainingspraxis auf Basis von Präsenzschulungen abschneidet. Die Ergebnisse zeigen, dass die Effektivität des entwickelten VR-Trainings in Bezug auf die Fähigkeit der TeilnehmerInnen, die Aufgabe erfolgreich zu bewältigen, mit der des traditionellen Trainings vergleichbar ist. Es wurde auch festgestellt, dass es noch Raum für Verbesserungen gibt (z. B. bei der Umsetzung der Interaktionen mit physischen Werkzeugen und Geräten sowie bei der Nachahmung und Verstärkung von Aspekten der sozialen Dynamik zwischen AusbilderIn und Auszubildendem). Saunders et al. (2019) berichten in einer ähnlichen Studie, dass VR-basierte Trainings, entweder allein oder in Kombination mit traditionellem hands-on training, genauso effektiv sein können wie ressourcenintensive klassische Trainingseinheiten.

Winther et al. (2020) präsentieren eine Fallstudie, in der eine VR-Trainingssimulation für Wartungsaufgaben einer Dosierpumpe mit traditionellen Trainingsmethoden (paarweises Training und Video-Training) verglichen wird. Untersucht wurden in dieser Studie Zeit pro Arbeitsschritt, Fehleranzahl und Bewertung der eigenen Handlungen. Die Ergebnisse zeigen, dass das VR-Training grundsätzlich zum Erlernen der Trainingsaufgaben geeignet war. Jedoch führten die beiden traditionellen Trainingsmethoden zu einer signifikanten Reduktion von Zeit und Fehleranzahl. Die AutorInnen führen die Ergebnisse darauf zurück, dass VR die Interaktion mit physischen Objekten nur unzureichend simulieren kann und VR-Training den Lernenden kognitiv zu wenig fordere.

Eine Gesamtschau der vorgestellten Befunde zeigt, dass das Potenzial von VR als Grundlage industrieller Trainings unklar ist. In den vorgestellten Studien werden weitere Forschungsarbeiten empfohlen. Die im vorliegenden Beitrag vorgestellte Studie soll einen Beitrag zum Erkenntnisfortschritt leisten. Basierend auf mehreren Vorprojekten im Bereich VR-gestütztes Training an der Fachhochschule Oberösterreich beschreiben wir im Folgenden die technische Implementierung eines Prototyps für immersives Training im Kontext komplexer Montageprozesse, der die Grundlage der hier berichteten empirischen Studie war. 


\section{Prototyp einer VR-Trainingsapplikation}

Die in unserer Laborstudie verwendete Applikation basiert auf einer Vorarbeit an der Fachhochschule Oberösterreich namens VRSmart. Diese Anwendung für gängige VR-Headsets (z.B. HTC Vive Pro) wurde in Unity entwickelt und bietet Werkzeuge zur Visualisierung und Manipulation komplexer Industrie-CAD-Daten (vgl. Wolfartsberger et al. 2018). Unser Ziel war es, ein Werkzeug zu entwickeln, mit dem User schnell und intuitiv 3D-Modelle betrachten und sich ein Bild von der zugrunde liegenden Konstruktionshierarchie machen können. Die aktuelle Version beinhaltet folgende Features:

- das Betrachten und Verschieben von Objekten entsprechend ihrer Konstruktionshierarchie,

- eine Schritt-für-Schritt-Montageanleitung für ein vordefiniertes Szenario (Abb. 1a),

- eine virtuelle Explosionszeichnung des gesamten Objekts (Abb. 1b) und

- eine virtuelle Schnittebene (Abb. 1c).

Im geführten Montagemodus arbeitet der User schrittweise eine vordefinierte Montage ab. Jeder Schritt im Montageprozess besteht aus mindestens einer Aktion, die der User ausführen muss, um zum nächsten Schritt zu gelangen (z. B. ein Objekt an die Zielposition verschieben oder ein Werkzeug berühren). Wichtige Hinweise werden automatisch angezeigt, um sicherzustellen, dass der User auf kritische Informationen achtet.

Grundsätzlich wird jedes Bauteil bzw. jedes Werkzeug, das für den aktuellen Schritt relevant ist, visuell hervorgehoben (siehe grüner Bereich in Abb. 1a). Bei Tätigkeiten, in denen ein Teil an einer bestimmten Position platziert werden muss, wird die Zielposition farblich hervorgehoben (siehe Abb. 2a, grüner Bereich). Wird das Bauteil in unmittelbarer Nähe der Zielposition platziert, „schnappt“ es automatisch ein (snapping) und gilt als korrekt montiert. Zusätzlich kann der User visuelle Hinweise (hints) anfordern, um Bauteile (und ihre Zielposition) zu markieren. Diese Hinweise sind nicht immer notwendig, haben sich aber bei komplexen Arbeitsschritten mit kleinen oder verdeckten Bauteilen als nützlich erwiesen.

Die Arbeitsschritte werden in einem Autorentool definiert, das eine zügige Erstellung neuer Montageszenarien ohne Programmierkenntnisse ermöglicht. Aktionen können hinzugefügt, umsortiert oder gelöscht werden. Bei Schritten, die beispielsweise das Platzieren eines Objekts beinhalten, werden Start- und Zielposition
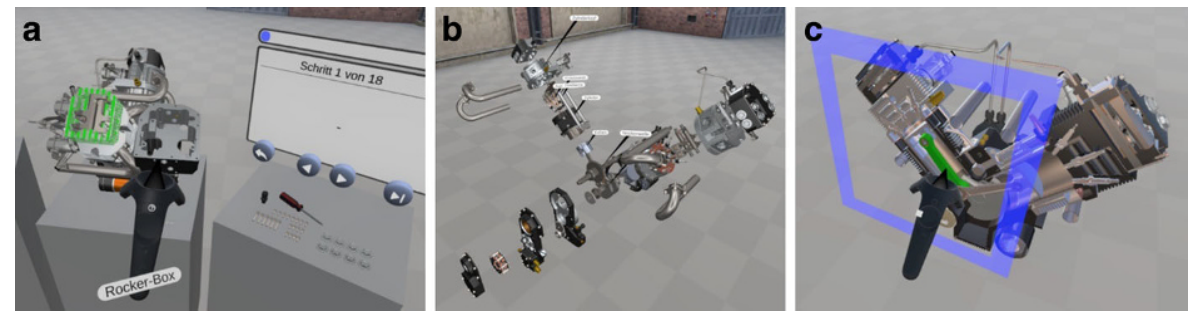

Abb. 1 Montagevorgang mit textuellen und visuellen Hinweisen (a); Explosionsansicht des Modells zur Ansicht einzelner Bauteile (b); Schnittansicht zur Visualisierung der inneren Struktur der Baugruppe (c) 

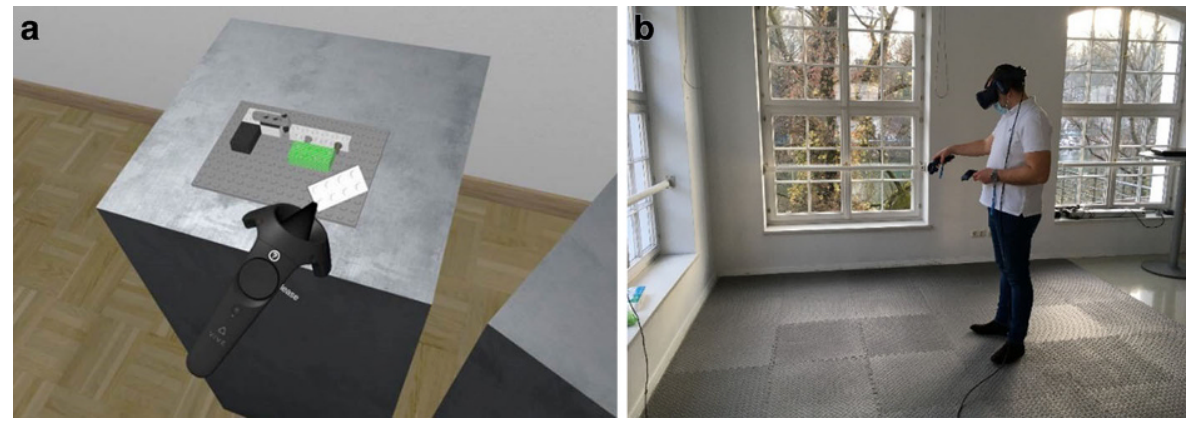

Abb. 2 Montage eines fiktiven Use Cases (a); die finale Position des Bauteils wird visuell hervorgehoben. Das Bauteil gilt als richtig montiert, wenn es in unmittelbarer Nähe der finalen Position platziert wird; VR User mit HTC Vive Pro beim virtuellen Training (b)

definiert. Für jeden Schritt kann der/die AutorIn zudem textuelle Anweisungen hinzufügen.

\section{Laborstudie}

Um Erkenntnisse über das Potenzial des VR-Trainingstools zur Wissensvermittlung zu gewinnen, wurde eine Laborstudie mit 24 ProbandInnen durchgeführt. Im Folgenden werden Methodik, Durchführung sowie Ergebnisse der Studie beschrieben.

\subsection{Versuchsaufbau und Methodik}

Auf der Basis eines Between-Subjects-Ansatzes wurden die TeilnehmerInnen in zwei Gruppen eingeteilt. Die Kontrollgruppe $(N=11)$ trainierte den fiktiven Montageprozess angeleitet von einem Tutor am realen Objekt. Die Experimentalgruppe $(N=13)$ durchlief das Training im VR-Trainingstool, angeleitet von visuellen Hinweisen in der 3D-Umgebung (Abb. 2). $\mathrm{Zu}$ montieren war ein Lego-Konstrukt bestehend aus 13 Arbeitsschritten (siehe Abb. 3). Wir haben uns bewusst für ein einfaches Szenario mit Lego-Bausteinen entschieden, da hierfür kein Vorwissen nö-

Abb. 3 Montageobjekt bestehend aus 13 Montageschritten

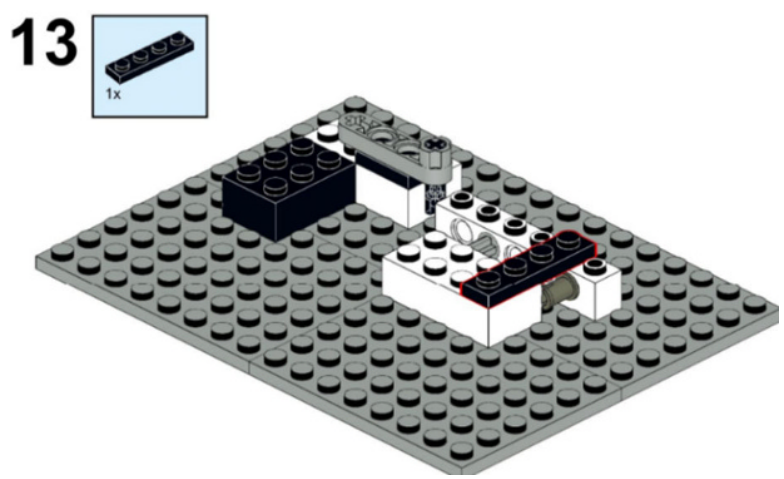


tig ist. Nach dem Trainingsdurchgang gab es eine 10-minütige Pause, in der die ProbandInnen bewusst abgelenkt wurden. Danach musste das Objekt noch einmal aus dem Gedächtnis aufgebaut werden. In diesem Durchgang wurden Montagezeit pro Arbeitsschritt, Fehlerrate sowie die Anzahl benötigter Hinweise gemessen. In beiden Durchgängen wurde die kognitive Belastung der Tätigkeiten mittels NASATLX Fragebogen erhoben (Hart 2006).

\subsection{Durchführung}

Die Einteilung der ProbandInnen in Kontroll- und Experimentalgruppe wurde nach dem Zufallsprinzip durchgeführt. Nach einer Begrüßung und einer Vorstellung der Aufgabe wurde eine Einwilligungserklärung und ein Fragebogen zur Erhebung von Alter, Geschlecht und Vorerfahrung mit VR ausgehändigt.

TeilnehmerInnen der Kontrollgruppe wurden nun zum Montagetisch geführt. Die Anordnung der Lego-Bausteine zu Beginn war vorgegeben. Für die gesamte Abfolge wurde im Vorfeld ein Skript angefertigt, das den TeilnehmerInnen Schritt für Schritt vorgelesen wurde. Bei Unklarheiten wurde die Anweisung erneut vorgelesen. Die TeilnehmerInnen der Experimentalgruppe konnten sich zunächst mit der VRHardware (HTC Vive Pro) vertraut machen. Nach einer kurzen Einführung in die allgemeinen Bedienungskonzepte konnten die Interaktionen am virtuellen Modell eines Motors geübt werden. Dazu zählten das korrekte Greifen, Verschieben und Platzieren von Objekten im virtuellen Raum. Nach der Eingewöhnung wurde die eigentliche Montage gestartet. Abb. 2 zeigt das Szenario: Der Baustein, der für den aktuellen Arbeitsschritt genommen werden musste, wurde farblich hervorgehoben. Nach dem Greifen des Bauteils wurde die Zielposition farblich markiert. In der virtuellen Umgebung existierten weder gesprochene noch textuelle Anweisungen. Der User folgte ausschließlich den visuellen Hinweisen des Systems.

Nach Durchführung der 13 Montageschritte wurde die individuelle Belastung während des Zusammenbaues des Legomodells mit einem standardisierten Fragebogen erhoben (NASA-TLX). Direkt im Anschluss mussten die ProbandInnen ein Merkspiel absolvieren, in dem sie sich neun Begriffe innerhalb von 30s merken mussten. Auf diese Weise wurde das Kurzzeitgedächtnis mit anderen Inhalten befüllt. Die Ergebnisse des Merkspiels waren für die Auswertung der Ergebnisse irrelevant.

Im zweiten Durchgang wurden die TeilnehmerInnen beider Gruppen zum Montagetisch geführt und mussten die 13 Arbeitsschritte frei aus dem Gedächtnis wiederholen. Dieser Durchgang wurde mithilfe einer Kamera gefilmt, um die Montagedauer und Fehler auswerten zu können. Eine weitere Person im Raum notierte Beobachtungen und besondere Vorkommnisse. Wenn der/die ProbandIn nicht weiterwusste, konnte von dieser Person ein Hinweis angefordert werden. Hinweise wurden der Kontrollgruppe in Form des standardisierten Skriptes aus dem ersten Durchgang vorgelesen. Es wurden keine Hinweise zur Richtigkeit des bisher aufgebauten Modells gegeben. Für die gesamte Montage wurden Montagezeit, Fehler und Anzahl benötigter Hinweise gemessen.

Es gab eine Unterscheidung von zwei Fehlertypen: Bausteinfehler traten auf, wenn die korrekte Reihenfolge der Montage nicht eingehalten wurde. Ein Baustein 
war also verbaut, obwohl ein Schritt zuvor noch nicht ausgeführt wurde. Der Fehler zählte, sobald der Baustein fest angebaut war und später auch nicht korrigiert wurde. Bei einem Positionsfehler erfolgte die Montage des Bausteins an der falschen Stelle. Ein Baustein, der an der falschen Position auf der Grundplatte montiert wurde, war als Positionsfehler vermerkt. Kein weiterer Fehler wurde gewertet, wenn auf diesem (falsch platzierten) Baustein ein weiterer Baustein laut Anleitung richtig montiert war. Nach dem zweiten Durchgang erfolgte die Messung der individuellen Belastung wieder mittels NASA-TLX. Danach wurden die TeilnehmerInnnen verabschiedet.

\subsection{Ergebnisse}

Im Folgenden werden die Ergebnisse dargestellt. Es nahmen 11 Frauen und 13 Männer an der Studie teil (21-57 Jahre, $M=35,4 ; S D=10,9)$. Die Hälfte hat zuvor schon ein VR-Headset benutzt. Die meisten TeilnehmerInnen gaben an, wenig Erfahrung mit VR zu haben $(\mathrm{M}=1,33 ; \mathrm{SD}=0,65 ; 1=$ wenig, $2=$ etwas, $3=$ viel $)$. Ein Großteil der Befragten spielt selten bis nie Computerspiele. Die Gruppe hatte also tendenziell wenig Vorwissen zu Interaktion und Navigation in virtuellen Welten. Zwischen der Experimental- und Kontrollgruppe bestanden hinsichtlich der genannten Variablen keine signifikanten Unterschiede.

Für den Vergleich der subjektiv empfundenen Belastung im Versuchsverlauf wurde der NASA-TLX-Fragebogen ausgewertet (siehe Abb. 4). Der Wertebereich von NASA-TLX ist: 0 (niedrig) bis 60 (hoch). Bei der Auswertung der Gesamtbelastung für den 1. Durchgang (training-on-the-job versus VR-Training) zeigt sich, dass sich bei der Kontrollgruppe eine durchschnittliche Gesamtbelastung von 22,95 Punkten ergibt $(\mathrm{SD}=4,83)$. Bei der Experimentalgruppe liegt dieser Wert bei 17,04 Punkten $(\mathrm{SD}=6,22)$. Somit haben die TeilnehmerInnen das Training in VR als kognitiv weniger anstrengend empfunden als das training-on-the-job mit dem Tutor ( $\mathrm{t}(22)=2,56$, $p<0,05)$. Wie in der Diskussion in Kap. 5 noch näher ausgeführt, wurde die Kontrollgruppe im training-on-the-job bereits kognitiv stärker gefordert. Die verbalen Anweisungen mussten verarbeitet und umgesetzt werden. Dies ist eine mögliche Erklärung, warum das VR-Training als weniger belastend empfunden wurde, es fehlte hier die kognitive Verarbeitung von Anweisungen.

Bei der Auswertung der Gesamtbelastung für den zweiten Durchgang zeigt sich, dass sich bei der Kontrollgruppe eine Gesamtbelastung von 31,00 ergibt (SD=6,47). Bei der Experimentalgruppe liegt dieser Wert bei 28,88 (SD=11,91). Somit haben die ProbandInnenen der Experimentalgruppe auch den zweiten Durchgang weniger anstrengend empfunden als die ProbandInnen der Kontrollgruppe. Der Unterschied ist jedoch nicht signifikant ( $(22)=0,53, p=0,604)$. Die Steigerungen der Werte vom ersten auf den zweiten Durchgang sind in beiden Gruppen statistisch signifikant (within-subjects ANOVA, $p<0,01$ ).

Im zweiten Durchgang mussten die ProbandInnen das Modell aus dem Gedächtnis erneut aufbauen. In einer späteren Auswertung der Videos wurden pro TeilnehmerIn folgende Parameter erhoben: Montagezeit, Bausteinfehler, Positionsfehler und Anzahl der Hinweise, die während des Montagevorganges von einer Drittperson eingeholt wurden. Tab. 1 zeigt eine Zusammenfassung der Ergebnisse. 
Abb. 4 Auswertung NASATLX zur mittleren Gesamtbelastung für beide Durchgänge, blau: Kontrollgruppe (trainingon-the-job), orange: Experimentalgruppe (VR-Training)
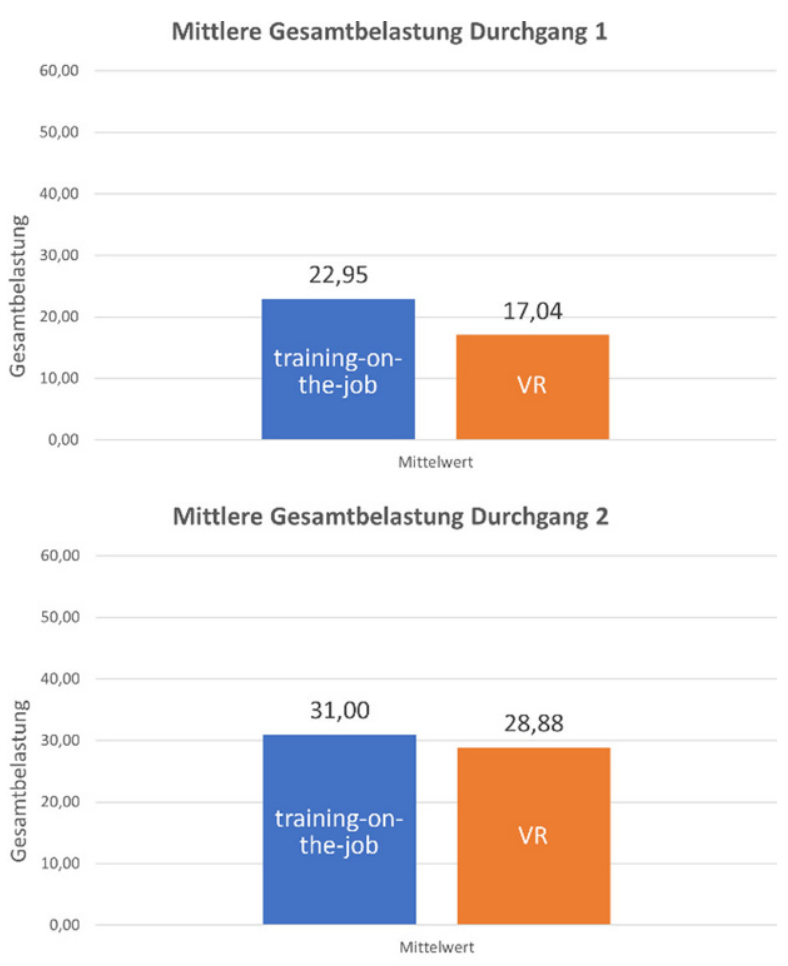

Die TeilnehmerInnen der Experimentalgruppe (VR-Training) haben hinsichtlich Fehlerhäufigkeit und Anzahl der Hinweise schlechtere Leistungen erzielt als die Kontrollgruppe. In Bezug auf Bausteinfehler (falscher Baustein oder falsche Reihenfolge) haben TeilnehmerInnen der Kontrollgruppe im Schnitt 2,09 Fehler gemacht $(\mathrm{SD}=3,24)$. Bei der Experimentalgruppe ist dieser Wert 3,46 $(\mathrm{SD}=3,23)$. Der Unterschied ist nicht signifikant ( $(22)=-1,034, p=0,312)$. Ein ähnliches Bild zeigt sich bei den Positionsfehlern (falsch platzierte Bausteine). Hier haben TeilnehmerInnen der Kontrollgruppe im Schnitt 3,09 Fehler gemacht (SD=3,62). Bei der Experimentalgruppe liegt der Wert bei 4,77 ( $\mathrm{SD}=3,63)$. Der Unterschied ist ebenso nicht signifikant $(\mathrm{t}(22)=-1,130, p=0,271)$. Obwohl die Ergebnisse statistisch nicht signifikant sind, zeigt sich der Trend, dass traditionelles Training für industrielle Montageabläufe in Summe bessere Ergebnisse erzielen konnte. Da die Wahrscheinlichkeit für einen statistisch signifikanten Unterschied zwischen zwei Gruppen mit zunehmen-

Tab. 1 Übersicht der Ergebnisse für Kontrollgruppe (KG, training-on-the-job) und Experimentalgruppe (EG, VR-Training)

\begin{tabular}{lllll}
\hline Gruppe & Zeit & Fehler (Baustein) & Fehler (Position) & Hinweise \\
\hline KG & $\mathrm{M}=3: 34 \mathrm{Min}$ & $\mathrm{M}=2,09$ & $\mathrm{M}=3,09$ & $\mathrm{M}=2,55$ \\
& $\mathrm{SD}=1: 51 \mathrm{Min}$ & $\mathrm{SD}=3,24$ & $\mathrm{SD}=3,62$ & $\mathrm{SD}=3,62$ \\
EG & $\mathrm{M}=4: 16 \mathrm{Min}$ & $\mathrm{M}=3,46$ & $\mathrm{M}=4,77$ & $\mathrm{M}=7,00$ \\
& $\mathrm{SD}=2: 06 \mathrm{Min}$ & $\mathrm{SD}=3,23$ & $\mathrm{SD}=3,63$ & $\mathrm{SD}=6,00$ \\
\hline
\end{tabular}




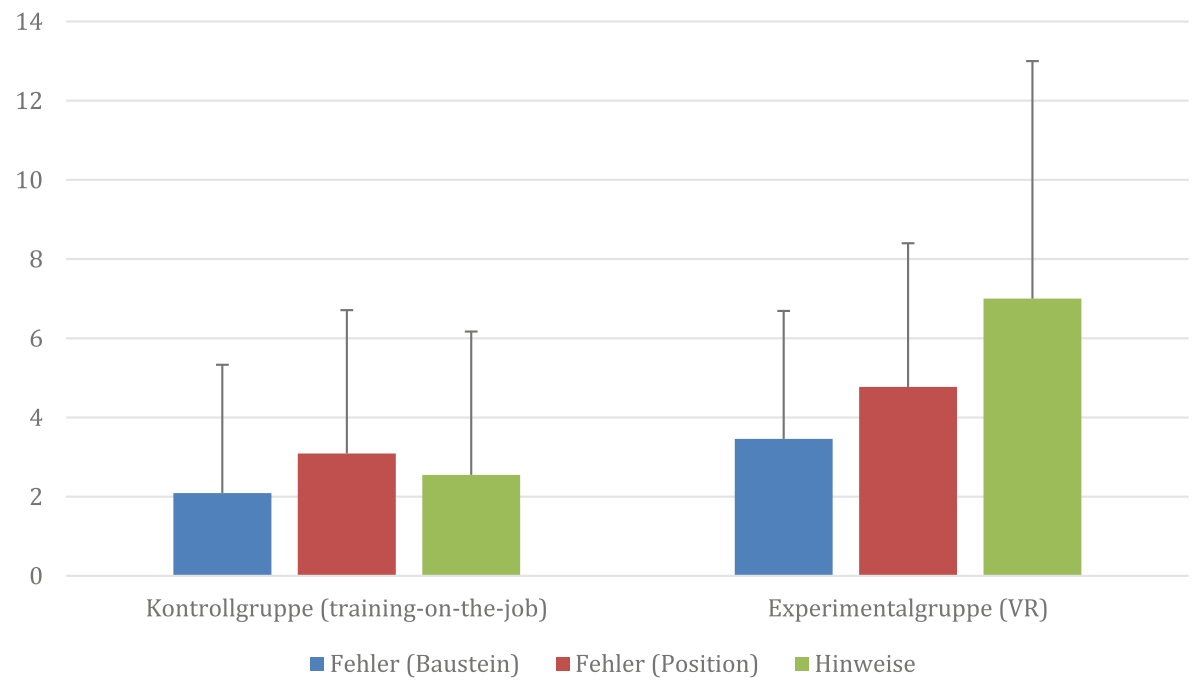

Abb. 5 Auswertung der Performancedimensionen nach dem zweiten Durchgang

der Stichprobengröße steigt, sollte zukünftige Forschung mit größeren Stichproben diese gefundene Tendenz weiter untersuchen.

Die TeilnehmerInnen der Experimentalgruppe benötigten im zweiten Durchgang bei 13 Arbeitsschritten im Schnitt sieben Hinweise, um die Montage zu beenden $(\mathrm{SD}=6,00)$. Dieser Wert war bei den TeilnehmerInnen der Kontrollgruppe mit durchschnittlich 2,55 Hinweisen deutlich niedriger $(\mathrm{SD}=3,62)$. Der Unterschied ist signifikant $(\mathrm{t}(15,2)=-2,507, p<0,05)$. VR-Training geht somit mit schlechterer Erinnerung an den Montagevorgang einher. Abb. 5 fasst die Ergebnisse zu Fehlerhäufigkeit und Anzahl der Hinweise für Kontroll- und Experimentalgruppe zusammen.

In Bezug auf Montagezeit benötigte die Kontrollgruppe im Schnitt 3 min und $34 \mathrm{~s}$ für die 13 Arbeitsschritte ( $\mathrm{SD}=1,51 \mathrm{~min})$. Die Experimentalgruppe benötigte hierfür mit 4 min und $16 \mathrm{~s}$ etwas länger $(\mathrm{SD}=2,06 \mathrm{~min})$.

\section{Diskussion der Ergebnisse}

Die Ergebnisse der Studie zeigen, dass VR-Training mit unserer Applikation weniger Wissen vermitteln konnte als das traditionelle Training, das von einem Tutor begleitet wird. Diese Erkenntnisse gehen mit aktuellen Befunden anderer Forschergruppen einher (vgl. Winther et al. 2020). Mögliche Ursachen für die gefundenen Ergebnisse werden im Folgenden diskutiert.

\subsection{Realismus der Interaktionen}

Die vorgestellte VR-Applikation bietet die Möglichkeit, einfache Montagetätigkeiten zu trainieren. Derzeit können Abfolgen von Tätigkeiten sowie Werkzeuggebrauch und textuelle Sicherheitshinweise integriert werden. Haptisches Feedback (zum Bei- 
spiel beim Berühren eines Bauteils) erfolgt lediglich durch Vibration der Controller. Sensorische Eindrücke wie Gewicht oder Materialbeschaffenheit können nach heutigem Technologiestand nicht realitätsgetreu in die Simulation integriert werden. Gerade hier gehen wertvolle Informationen verloren, die zum Lernerfolg beitragen können.

Studien zeigen, dass die parallele Beteiligung verschiedener Sinne den Lernprozess signifikant unterstützen kann (Shams and Seitz 2008; Sénécal et al. 2013). Beispielsweise zeigen Forschungsbefunde von Gavish et al. (2015), dass Interaktionsmodi, welche die Beteiligung verschiedener Sinne implizieren (z.B. haptisches Feedback), einen wesentlichen Beitrag zur Erklärung von Unterschieden in Ergebnisvariablen (z. B. Bearbeitungszeit, Fehlerrate) leisten können. Ähnliche Ergebnisse werden in weiteren Studien berichtet (z. B. Makransky et al. 2019). Im gegenständlichen Fall ist es wahrscheinlich, dass durch die vereinfachte Darstellung einzelner Arbeitsschritte die User in der VR-Bedingung kognitiv zu wenig gefordert wurden. Um den Realismus und den Lerneffekt des VR-Trainings zu erhöhen, sollten daher künftig neue Möglichkeiten der Interaktion erforscht werden. Erste Ansätze liefern Force Feedback-Systeme zur Simulation von Gewicht und Widerstand und HandTracking als Ersatz von Controllern (Choi et al. 2017).

\subsection{Detailgrad der Hilfestellungen}

In der aktuellen Version der VR-Trainingsapplikation wird jeder Arbeitsschritt durch visuelle Hinweise unterstützt. Dieser hohe Detailgrad an Hinweisen kann die User davon abhalten, kognitiv gefordert zu sein, weil pure wiederholte Informationsaufnahme an die Stelle des Ziehens logischer Schlüsse tritt. Unsere Beobachtungen haben gezeigt, dass User die Vorgaben des VR-Systems umgesetzt haben, ohne über die korrekte Durchführung der Arbeitsschritte nachzudenken. Anders formuliert: Das System gibt jeden Handgriff vor. Der User arbeitet die Schritte ab, muss jedoch nie über mögliche Alternativen nachdenken. Ein User meinte im Feedback nach der Test Session: „Es ist vergleichbar mit einem Navigationssystem im Auto. Die Technologie bringt dich garantiert ans Ziel, von der Geographie und möglichen Streckenalternativen lernt man aber nichts." Um den Lernerfolg nachhaltig zu erhöhen, müssen VR-Trainings deutlich mehr Handlungsalternativen bieten. Zukünftige Forschungsarbeiten könnten die Verwendung verschiedener Detailgrade virtueller Handlungsanweisungen untersuchen und wie sich diese konkret auf den Lernerfolg auswirken. Dabei muss bedacht werden, dass nicht nur zu viel, sondern auch zu wenig Anleitung negative Auswirkungen auf die Akzeptanz haben können. Wenn der User nicht weiß, wie er im Training vorankommt, kann Frustration entstehen und sich die Lernbereitschaft verringern. Daher sollte die Möglichkeit bestehen, zusätzliche Informationen anzufordern. Winther et al. (2020) weisen darauf hin, dass es von Vorteil ist, redundante Informationen über mehrere Sinneskanäle anzubieten.

Ein Schwachpunkt unserer VR-Applikation (und auch vieler vergleichbarer Lösungen) besteht darin, dass während des Lernprozesses keine Fehler gemacht werden können. Die VR-Trainingssimulation erlaubt nur, die richtige Komponente zu wählen. Diese kann auch nur an der vorgesehenen (korrekten) Position abgelegt werden. Alternativen sind im Authoring-System unseres Prototypen derzeit nicht vorgesehen. 
Das führt dazu, dass der User nicht darüber nachdenken muss, mit welchem Werkzeug er im aktuellen Arbeitsschritt interagieren muss oder an welcher Position das Bauteil zu montierten ist. Im training-on-the-job, das von einem Tutor begleitet wird, können diese falsch ausgeführten Tätigkeiten sehr wohl auftreten. Unsere Beobachtungen in der Kontrollgruppe haben gezeigt, dass das regelmäßig passiert ist. Der/Die ProbandIn war dadurch gezwungen, sich aktiv mit dem Gelernten auseinanderzusetzen. Bauteile dürfen nicht automatisch „einrasten“, wenn Sie nur nahe genug an der finalen Position platziert werden. Zudem muss beim Training die Interaktion mit allen verfügbaren virtuellen Komponenten bzw. Bauteilen zu jederzeit möglich sein. In einer weiterführenden Studie wollen wir hier ansetzen und die Auswirkungen der visuellen Hilfestellungen auf den Lernerfolg in virtuellen Trainings untersuchen.

\section{Zusammenfassung und Ausblick}

In der vorliegenden Studie konnte VR-Training keinen besseren Lerneffekt erzielen als traditionelles training-on-the-job. Die Daten zeigen, dass die Kontrollgruppe in Bezug auf Montagefehler ein wenig und hinsichtlich Anzahl benötigter Hinweise im zweiten Durchgang sogar deutlich besser abschnitt. Die ProbandInnenen der VRGruppe empfanden den ersten Durchgang als kognitiv weniger belastend. Eine entscheidende Implikation unserer Studie für die Praxis ist daher, dass ein Unterschreiten des kognitiven Aufwands beim Lernen die Wirksamkeit des Trainings ungünstig beeinflussen kann. Konkret legen unsere Ergebnisse nahe, dass die vereinfachte Darstellung von Arbeitsschritten sowie fehlende multimodale Reize während des VRTrainings den Lerneffekt negativ beeinflussen. Hinsichtlich der Relevanz multimodaler Reize für Lern- und Gedächtnisprozesse hat die Forschung im Bereich der kognitiven Neurowissenschaften (z.B. Gallace und Spence 2009) sowie NeuroIS (z. B. Riedl und Léger 2016) bereits wertvolle Erkenntnisse erarbeitet. Sénécal et al. (2013) konnten beispielsweise auf der Basis der Elektroenzephalographie (EEG) zeigen, dass Lern- und Gedächtnisprozesse signifikant vom subjektiven Grad für ,need for touch“ (NFT) abhängig sein können (NFT ist definiert als ,,preference for the extraction and utilization of information obtained through the haptic system") (Peck und Childers 2003, S. 431).

Um die Ergebnisse der vorliegenden Studie zu bestätigen, müssen weitere Untersuchungen durchgeführt werden. Dies ist auch deshalb notwendig, weil die Stichprobe in der vorliegenden Laborstudie klein ist. Unsere Arbeit dient als Basis für weitere Untersuchungen, um neue Erkenntnisse über den Lerneffekt in VR-Trainings zu gewinnen. Ein NeuroIS-Ansatz auf der Basis eines Einsatzes neurophysiologischer Messverfahren und Theorien sollte dabei in Erwägung gezogen werden.

Obwohl das VR-System in dieser Studie im Vergleich zum traditionellen Training weniger Wissen vermitteln konnte (gemessen über die Fehlerhäufigkeit und Anzahl der benötigten Hinweise), ist das Potenzial von VR-Technologie zur nachhaltigen Wissensvermittlung grundsätzlich gegeben. Für die Praxis gilt es jedoch zu beachten, dass der bloße und unreflektierte Einsatz von VR-Technologie ohne Berücksichtigung seiner vielschichtigen Facetten (z.B. Zulassen von Fehlern, um daraus zu lernen) mit ungünstigen Wirkungen einhergehen kann. 
Danksagung Das Projekt Smart Factory Lab wird aus Mitteln des Europäischen Fonds für regionale Entwicklung (EFRE) und des Landes Oberösterreich kofinanziert.

Funding Open access funding provided by University of Applied Sciences Upper Austria.

Open Access Dieser Artikel wird unter der Creative Commons Namensnennung 4.0 International Lizenz veröffentlicht, welche die Nutzung, Vervielfältigung, Bearbeitung, Verbreitung und Wiedergabe in jeglichem Medium und Format erlaubt, sofern Sie den/die ursprünglichen Autor(en) und die Quelle ordnungsgemäß nennen, einen Link zur Creative Commons Lizenz beifügen und angeben, ob Änderungen vorgenommen wurden.

Die in diesem Artikel enthaltenen Bilder und sonstiges Drittmaterial unterliegen ebenfalls der genannten Creative Commons Lizenz, sofern sich aus der Abbildungslegende nichts anderes ergibt. Sofern das betreffende Material nicht unter der genannten Creative Commons Lizenz steht und die betreffende Handlung nicht nach gesetzlichen Vorschriften erlaubt ist, ist für die oben aufgeführten Weiterverwendungen des Materials die Einwilligung des jeweiligen Rechteinhabers einzuholen.

Weitere Details zur Lizenz entnehmen Sie bitte der Lizenzinformation auf http://creativecommons.org/ licenses/by/4.0/deed.de.

\section{Literatur}

Abulrub A-HG, Attridge AN, Williams MA (2011) Virtual reality in engineering education: the future of creative learning. In: IEEE Global Engineering Education Conference (EDUCON). IEEE, Amman, S $751-757$

Choi I, Culbertson H, Miller MR, Olwal A, Follmer S (2017) Grabity: a wearable Haptic interface for simulating weight and grasping in virtual reality. In: Proceedings of the 30th annual ACM symposium on user interface software and technology (UIST '17). ACM, New York, S 119-130

Cmentowski S, Krekhov A, Krüger J (2019) Outstanding: a multi-perspective travel approach for virtual reality games. In: Proceedings of the annual symposium on computer-human interaction in play (CHI PLAY '19). ACM, New York, S 287-299

Fromm J, Radianti J, Wehking C, Stieglitz S, Majchrzak TA, von Brocke J (2021) More than experience?-On the unique opportunities of virtual reality to afford a holistic experiential learning cycle. Internet High Educ 50:1-14

Gallace A, Spence C (2009) The cognitive and neural correlates of tactile memory. Psychol Bull 135(3):380-406

Gavish N, Gutiérrez T, Webel S, Rodríguez J, Peveri M, Bockholt U, Tecchia F (2015) Evaluating virtual reality and augmented reality training for industrial maintenance and assembly tasks. Interact Learn Environ 23(6):778-798

Grassini S, Laumann K (2020) Evaluating the use of virtual reality in work safety: a literature review. In: Proceedings of the 30th European safety and reliability conference (ESREL) 01.11.-05.11.2020 Research Publishing Services, Singapore, S 4964-4971

Hart SG (2006) Nasa-task load index (NASA-TLX); 20 years later. Proc Hum Factors Ergon Soc Annu Meet 50(9):904-908

Huang W, Roscoe RD, Johnson-Glenberg MC, Craig SD (2021) Motivation, engagement, and performance across multiple virtual reality sessions and levels of immersion. J Comput Assist Learn 37:745-758

Makransky G, Borre-Gude S, Mayer RE (2019) Motivational and cognitive benefits of training in immersive virtual reality based on multiple assessments. J Comput Assist Learn 35(6):691-707

Mulders M (2020) Investigating learners' motivation towards a virtual reality learning environment: a pilot study in vehicle painting. In: International Conference on Artificial Intelligence and Virtual Reality (AIVR) 14.12.-18.12.2020 IEEE, Utrecht, S 390-393

Peck J, Childers TL (2003) To have and to hold: the influence of haptic information on product judgments. J Mark 67(2):35-48

Pirker J, Lesjak I, Parger M, Gütl C (2018) An educational physics laboratory in mobile versus room scale virtual reality - a comparative study. In: Online engineering \& internet of things. Lecture Notes in Networks and Systems, Bd. 22. Springer, Cham, S 1029-1043

Pratticò FG, Lamberti F (2021) Towards the adoption of virtual reality training systems for the self-tuition of industrial robot operators: a case study at KUKA. Comput Ind 129:1-11 
Riedl R, Léger P-M (2016) Fundamentals of NeuroIS—information systems and the brain. Springer, Berlin Heidelberg

Saunders J, Davey S, Bayerl PS, Lohrmann P (2019) Validating virtual reality as an effective training medium in the security domain. In: IEEE conference on virtual reality and 3D user interfaces 23.03.-27. 03.2019 IEEE, Osaka, S 1908-1911

Sénécal S, Léger P-M, Fredette M, Courtemanche F, Cameron A-F, Mirhoseini S, Paquet A, Riedl R (2013) Mouse vs. Touch screen as input device: does it influence memory retrieval? In: International conference on information systems (ICIS 2013): reshaping society through information systems design. 5

Shams L, Seitz AR (2008) Benefits of multisensory learning. Trends Cogn Sci 12(11):411-417

Winther F, Linoj R, Svendsen KP, Feuchtner T (2020) Design and evaluation of a VR training simulation for pump maintenance based on a use case at Grundfos. In: IEEE conference on virtual reality and 3D user interfaces 22.03.-26.03.2020 IEEE, Atlanta, S 738-746

Wolfartsberger J, Zenisek J, Sievi C (2018) Chances and Limitations of a Virtual Reality-supported Tool for Decision Making in Industrial Engineering. IFAC-PapersOnLine 51(11):637-642

Zenisek J, Wild N, Wolfartsberger J (2021) Investigating the Potential of Smart Manufacturing Technologies. Procedia Computer Science 180:507-516 